\title{
ALTERACIONES PROSTÁTICAS EN CANINOS DETERMINADAS MEDIANTE ULTRASONOGRAFÍA Y CITOLOGÍA POR ASPIRACIÓN ECO-GUIADA
}

\author{
Prostatic Disorders in Canines determined by Ultrasound Examination \\ AND ECo-Guided AsPiration CyTOLOGY
}

Susan Peña N. ${ }^{1,3}$, Alfonso Chavera C. ${ }^{2}$, Jacqueline Cahua U. ${ }^{1}$, Rosa Perales C. ${ }^{2,4}$

\section{Resumen}

\begin{abstract}
El objetivo del presente trabajo fue determinar la frecuencia y tipo de alteraciones prostáticas en caninos diagnosticadas a través de ultrasonografía y citología. Se trabajó con 62 caninos no castrados, pacientes de la Clínica de Animales Menores de la Facultad de Medicina Veterinaria de la Universidad Nacional Mayor de San Marcos, Lima. Se realizó el examen ecográfico y luego se hizo la aspiración prostática con aguja fina guiada por ecografía. Las láminas fueron coloreadas con Tinción-15 y se hizo el reconocimiento celular. Se evaluó el efecto del grupo etario, tamaño, raza y signos clínicos en relación al tipo de alteración prostática. Se determinó que 40 animales $(64.5 \%)$ presentaron alguna patología prostática diagnosticada mediante alguna de las dos pruebas. Con la técnica de ultrasonografía se detectaron quistes o abscesos (4.8\%), hiperplasia prostática (32.3\%), hiperplasia y prostatitis (20.9\%), prostatitis (4.8\%) y neoplasias (1.6\%); en tanto que por citología se determinó hiperplasia glandular (32.3\%), hiperplasia asociada a proceso inflamatorio $(16.1 \%)$, prostatitis $(12.9 \%)$ y adenocarcinoma (3.2\%). Se encontró asociación estadística de la edad y los signos clínicos con la presencia de alguna patología prostática $(\mathrm{p}<0.05)$; asimismo, se determinó concordancia entre los diagnósticos con las dos pruebas ( $\mathrm{p}<0.05)$, siendo menor la correlación con los casos de inflamación prostática $(\mathrm{k}=0.315)$.
\end{abstract}

Palabras clave: caninos, próstata, alteración, aspiración, ultrasonografía, citología

\section{Abstract}

The aim of this study was to determine the frequency and type of prostate disorders in canines through the use of ultrasound and cytology techniques. A total of 62 noncastrated dogs that were patients of the Small Animals Clinic of the Veterinary Faculty of San Marcos University, Lima were used. The ultrasound examination was conducted followed by the ultrasound-guided prostatic aspiration technique. The smears were stained and the cell recognition was done. The effect of age, size, breed and clinical signs

\footnotetext{
${ }^{1}$ Clínica de Animales Menores, ${ }^{2}$ Laboratorio de Histología, Embriología y Patología Veterinaria, Facultad de Medicina Veterinaria, Universidad Nacional Mayor de San Marcos, Lima

${ }^{3}$ E-mail:merrika_1201@hotmail.com,merrika.1201@gmail.com

${ }^{4}$ E-mail: rperales_fmv@hotmail.com
} 
on the clinical disorder was evaluated. The results showed that 40 animals $(64.5 \%)$ had a prostate disorder with any of the two tests. The ultrasound technique showed cysts or abscesses $(4.8 \%)$, hyperplasia $(32.3 \%)$, hyperplasia and prostatitis $(20.9 \%)$, prostatitis (4.8\%) and neoplasias $(1.6 \%)$; while through cytology was found glandular hyperplasia $(32.3 \%)$, hyperplasia associated to inflammation (16.1\%), prostatitis $(12.9 \%)$ and adenocarcinoma $(3.2 \%)$. Statistical association was found between age and clinical signs with the presence of prostatic disorders $(\mathrm{p}<0.05)$. Also, there was statistical concordance between the diagnose resulting from the two tests $(\mathrm{p}<0.05)$, however the correlation was lower in cases of prostatic inflammation $(\mathrm{k}=0.315)$.

Key words: dogs, prostate, alteration, aspiration, ultrasonography, cytology

\section{INTRODUCCIÓN}

La próstata aporta parte de los líquidos seminales durante la eyaculación, lo cual facilita el transporte y supervivencia de los espermatozoides en la monta natural (García, 1996). En el caso del canino, la próstata es la única glándula sexual accesoria, se encuentra bastante desarrollada y se localiza sobre el cuello de la vejiga y en posición ventral al recto.

El perro sufre de patologías prostáticas conforme avanza la edad; asimismo, las razas medianas y grandes son más propensas a desarrollar este tipo de problemas, siendo el Doberman Pinscher y el Pastor Alemán las más afectadas. Los principales trastornos que afectan a la próstata son la hiperplasia benigna, metaplasia escamosa, inflamación, formación de quistes y abscesos, quistes paraprostáticos y neoplasias (Paclikova et al., 2006).

Existen diversos síndromes de enfermedad prostática que comparten signos clínicos semejantes como tenesmo, hematuria, disuria y descarga uretral. Para su diferenciación, es necesario realizar la anamnesis junto al examen clínico como paso previo a las pruebas diagnósticas (Senior, 2006), entre los que se encuentran la palpación rectal, examen de fluido prostático y semen, radiología, ultrasonografía, citología y biopsia prostática (Barsanti y Finco, 2002).
El uso de la ultrasonografía proporciona una información valiosa ya que permite obtener imágenes de la estructura interna, y ayuda en la selección de los procesos que podrían ser incluidos en el diagnóstico diferencial. Asimismo, facilita la obtención de muestras de la lesión mediante la aspiración con aguja fina y biopsia percutánea (Nyland y Mattoon, 2002). Por otro lado, la citología se utiliza también como técnica diagnóstica porque permite identificar el tipo, estructura y morfología de las células y sus elementos para poder evaluar procesos inflamatorios, proliferativos y neoplásicos (Gadelha et al., 2009).

El presente trabajo tuvo por objetivo determinar el tipo y frecuencia de alteraciones prostáticas en caninos mediante el uso de la ultrasonografía y citología por aspiración con aguja fina.

\section{Materiales y Métodos}

La toma de muestras se realizó en la Clínica de Animales Menores (CAM) y su procesamiento se hizo en el Laboratorio de Histología, Embriología y Patología Veterinaria, ambas de la Facultad de Medicina Veterinaria de la Universidad Nacional Mayor de San Marcos, Lima.

Se trabajó con 62 caninos machos enteros, mayores de dos años, seleccionados al 
azar dentro de los pacientes de la CAM, entre noviembre de 2009 a junio de 2010. Los propietarios de los animales que se incluyeron en el estudio dieron su consentimiento firmado. La información recolectada incluyó la raza, edad, tamaño, peso y presencia o ausencia de signos clínicos propios de enfermedades prostáticas. El tamaño de muestra fue obtenido con la fórmula de cuantificación de una proporción, tomando el $20 \%$ como frecuencia esperada (Avendaño, 2008).

Se hizo un examen ultrasonográfico de la próstata utilizando un ecógrafo Sonoace Pico con transductor microconvexo de 4 a 9 MHz. Se determinó la situación, tamaño, forma, estructura capsular y características del parénquima prostático. Se consideraron como valores longitudinales normales los siguientes: perros con pesos menores de $8 \mathrm{~kg}$ de $2.14 \pm 0.46 \mathrm{~cm}$, perros de 8 a $18 \mathrm{~kg}$ de $2.90 \pm$ $0.98 \mathrm{~cm}$ y perros con pesos mayores de 18 $\mathrm{kg}$ de $3.44 \pm 0.72 \mathrm{~cm}$ (Miguel de Priego, 2007). Asimismo, se consideró una próstata de forma irregular a toda aquella que no fuese alargada en los cortes longitudinales y bilobulada y simétrica en los cortes transversales (Avendaño, 2008). Se registró la presencia de estructuras quísticas de contenido hipoecogénico o anecoico, así como ecogenicidad no isoecoica a otras zonas.

Para la aspiración guiada por ecografía, se preparó la zona prepúbica a punzar como un campo quirúrgico aséptico. En la punción se utilizaron agujas $21 \mathrm{G}$ x 11/2" acopladas a jeringas de 5, 10 o $20 \mathrm{ml}$. La imagen ecográfica fue utilizada para direccionar la aguja hacia la próstata y a las zonas en que se detectaban anormalidades ecográficas. Para la aspiración se realizaron varios movimientos circulares dentro del órgano sin extraer la aguja hasta obtener un adecuado volumen de muestra, y a su vez, el émbolo fue movido hacia arriba varias veces para realizar dicha aspiración (Meinkoth et al., 1999). Este procedimiento se realizó de dos a tres veces en diferentes áreas del parénquima prostático.
Se aplicaron tranquilizantes a los animales nerviosos o agresivos para el examen ultrasonográfico y toma de muestras para citología. Se usó maleato de acepromazina $(0.05 \mathrm{mg} / \mathrm{kg}$, IM o IV) o la asociación de clorhidrato de ketamina ( $2 \mathrm{mg} / \mathrm{kg}$, IV) y diazepam $(0.2 \mathrm{mg} / \mathrm{kg}, \mathrm{IV})$. Para el procedimiento, los animales fueron colocados en posición decúbito dorsal sobre una mesa con soporte en forma de $\mathrm{V}$, rasurándose la zona púbica de aquellos con pelaje denso.

La muestra obtenida se colocó en una lámina portaobjetos y se extendió con otra lámina. En el caso de haber obtenido abundante material, se dejaba sedimentar por dos horas para evaluar únicamente el sedimento. Las láminas fueron coloreadas con Tinción T-15 (Biopur Diagnostics, Argentina). Las láminas se evaluaron con microscopio óptico y con objetivos de $4 \mathrm{X}, 10 \mathrm{X}$ y $40 \mathrm{X}$.

Se estableció la frecuencia de presentación de alteraciones prostáticas. La asociación entre los resultados positivos y negativos a la presencia de enfermedad prostática y las variables edad, tamaño, raza y signos clínicos fue evaluada mediante la prueba de Chi cuadrado. Asimismo, se determinó la concordancia de los diagnósticos resultantes de los dos exámenes utilizados mediante la prueba de Kappa.

\section{Resultados}

Se encontró asociación estadística entre la edad y signos clínicos con la presencia de alteraciones prostáticas $(\mathrm{p}<0.05)$, habiendo una mayor ocurrencia en el grupo de canes mayores de 10 años (100\%) y de canes con signos clínicos (100\%); sin embargo, no se encontró asociación con las demás variables (Cuadro 1). El uso de los fármacos tranquilizantes solo fue necesario en 10 animales y ninguno presentó complicaciones posteriores por las punciones o por los medicamentos. 
Cuadro 1. Frecuencias de alteraciones prostáticas detectadas en caninos mediante ultrasonografía y citología, según edad, tamaño y peso, raza y signos clínicos

\begin{tabular}{ccccc}
\hline \multirow{2}{*}{ Variable } & Perros & \multicolumn{2}{c}{ Alteraciones } \\
\cline { 4 - 5 } & & $(\mathrm{n})$ & $\mathrm{N} .^{\circ}$ & $(\%)$ \\
\hline Edad (años) & & & \\
$<5$ & 15 & 5 & $33.3^{\mathrm{a}}$ \\
$5-<10$ & 29 & 17 & $58.6^{\mathrm{a}}$ \\
$>10$ & 18 & 18 & $100.0^{\mathrm{b}}$ \\
Tamaño y peso del animal (kg) & & & \\
Pequeños & $(<10)$ & 8 & 5 & $62.5^{\mathrm{a}}$ \\
Medianos & $(10.1-20)$ & 24 & 14 & $58.3^{\mathrm{a}}$ \\
Grandes & $(20.1-35)$ & 20 & 14 & $70.0^{\mathrm{a}}$ \\
Gigantes & $(>35)$ & 10 & 7 & $70.0^{\mathrm{a}}$ \\
Raza & & & & \\
Mestiza & & 25 & 14 & $56.0^{\mathrm{a}}$ \\
Pura & & 37 & 26 & $70.3^{\mathrm{a}}$ \\
Signos clínicos & & & & \\
Presentes & & 26 & 26 & $100.0^{\mathrm{a}}$ \\
Ausentes & & 62 & 14 & $38.9^{\mathrm{b}}$ \\
\hline
\end{tabular}

${ }^{a, b}$ Superíndices diferentes indican diferencias significativas $(p<0.05)$

Las principales alteraciones ecográficas encontradas fueron prostatomegalia, estructuras cavitarias, forma prostática irregular y variaciones en la ecogenicidad. En base a estas alteraciones se pudo establecer los diagnósticos clínicos (Cuadro 2).

En la hiperplasia, las próstatas presentaban una forma más ovalada, de bordes regulares, con ecogenicidad normal o aumentada y de mayor tamaño, donde una de ellas llegó a medir 84 mm de largo. En 10 de estos casos se encontraron estructuras cavitarias en el parénquima con contenido anecoico ( 9 / 10) o hipoecogénico $(1 / 10)$.

Se encontraron 13 casos con diagnóstico compatible con hiperplasia prostática y prostatitis, donde hubo aumento de tamaño y estructuras cavitarias con contenido anecoico (7/13) o hipoecogénico (6/13). Además, se observaron cinco casos con estructuras quísticas paraprostáticas en posición craneal a la vejiga de contenido anecoico (2/5) o hipoecogénico (3/5) (Fig. 1). 
Cuadro 2. Frecuencia de presentación de patologías prostáticas en caninos evaluados mediante ultrasonografía transabdominal

\begin{tabular}{lcc}
\hline \multirow{2}{*}{ Diagnóstico compatible } & \multicolumn{2}{c}{ Canes con alteraciones prostáticas } \\
\cline { 2 - 3 } & $\mathrm{n}$ & $\%$ \\
\hline Hiperplasia prostática & 20 & 32.3 \\
Hiperplasia y prostatitis & 13 & 20.9 \\
Prostatitis & 3 & 4.8 \\
Quistes o abscesos & 3 & 4.8 \\
Neoplasia & 1 & 1.6 \\
\hline
\end{tabular}

Cuadro 3. Frecuencia de presentación de patologías prostáticas en caninos evaluados mediante citología por aspiración con aguja fina guiada por ecografía

\begin{tabular}{lcc}
\hline & \multicolumn{2}{c}{ Canes con alteraciones prostáticas } \\
\cline { 2 - 3 } Diagnóstico compatible & $\mathrm{n}$ & $\%$ \\
\hline Hiperplasia glandular & 20 & 32.3 \\
$\begin{array}{l}\text { Hiperplasia glandular asociada a } \\
\text { proceso inflamatorio }\end{array}$ & 10 & 16.1 \\
Prostatitis & 8 & 12.9 \\
Adenocarcinoma prostático & 2 & 3.2 \\
\hline
\end{tabular}

Las alteraciones fueron consideradas como prostatitis cuando la glándula no estaba muy agrandada pero presentaba una disminución de la ecogenicidad con un carácter focal formando áreas hipoecogénicas y con estructuras quísticas. Los casos donde solo hubo estructuras cavitarias sin aumento de tamaño de la glándula ni cambios en la ecogenicidad del parénquima fueron considerados como quistes o abscesos. Asimismo, se diagnosticó un caso compatible con neoplasia prostática que se caracterizó por presentar asimetría del parénquima, bordes irregulares, ecogenicidad mixta, áreas cavitarias e hiperecogénicas y presencia de calcificaciones (Fig. 2).
Mediante la técnica de citología por aspiración guiada se logró diagnosticar 40 canes con patologías prostáticas, representando el $64.5 \%$ de los animales muestreados (Cuadro 3).

En la hiperplasia glandular se evidenció un aumento del número de células epiteliales prostáticas conservando sus características homogéneas, formando acinos de mayor tamaño y número; donde algunas células presentaban núcleos de forma ovalada y de posición excéntrica, y citoplasma granular con vacuolas de bordes nítidos en su interior.

Hubo 10 casos con diagnóstico de hiperplasia asociada a proceso inflamatorio, 


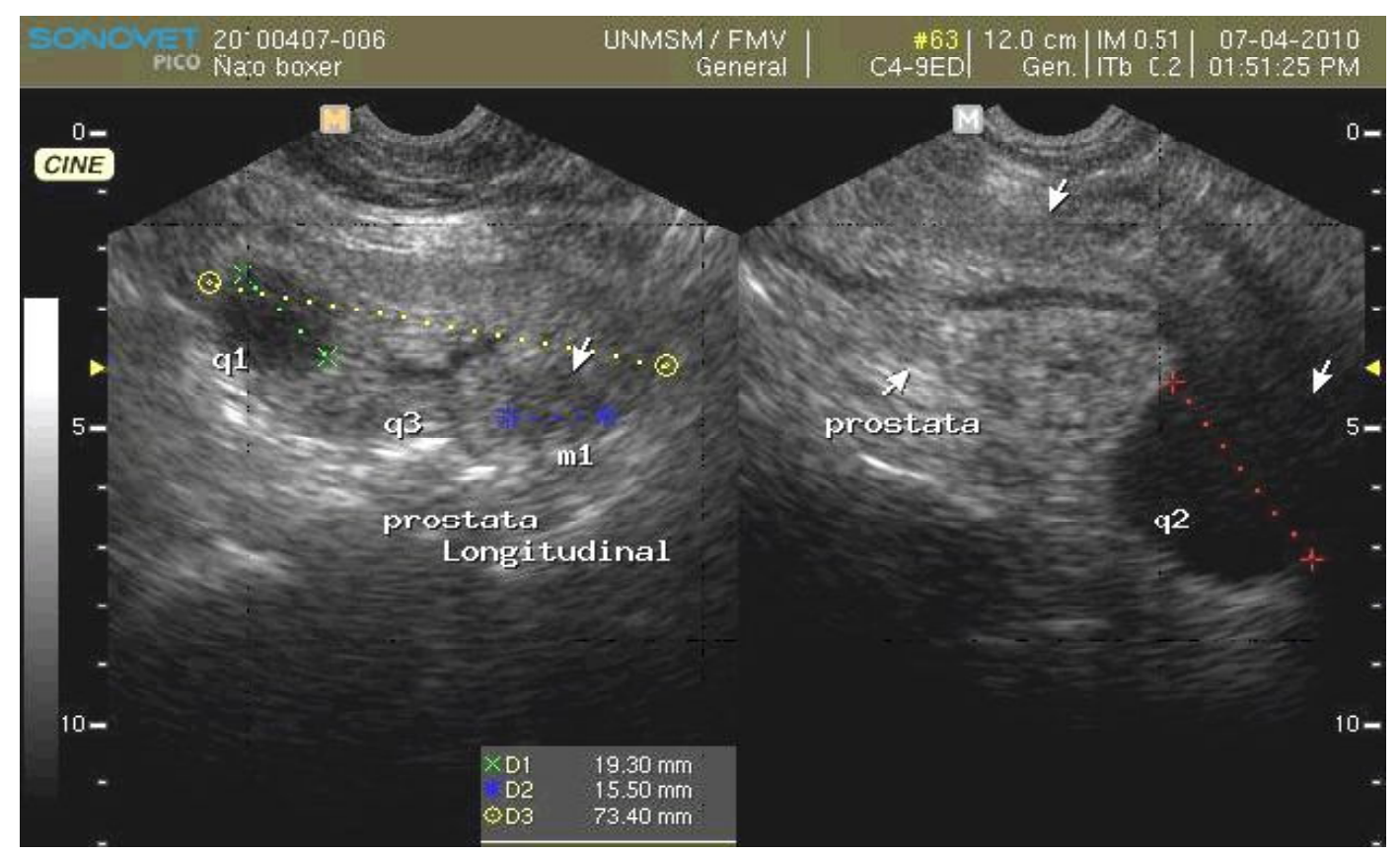

Figura 1. Lesiones compatibles con hiperplasia y prostatitis en un canino. Se observa el parénquima homogéneo pero con estructuras cavitarias de contenido anecoico de $19.3 \mathrm{~mm}$ (q1) y otra de $37.1 \mathrm{~mm}$ en la zona caudal (q2) y una área hipoecogénica irregular de $15.5 \mathrm{~mm}(\mathrm{m1})$

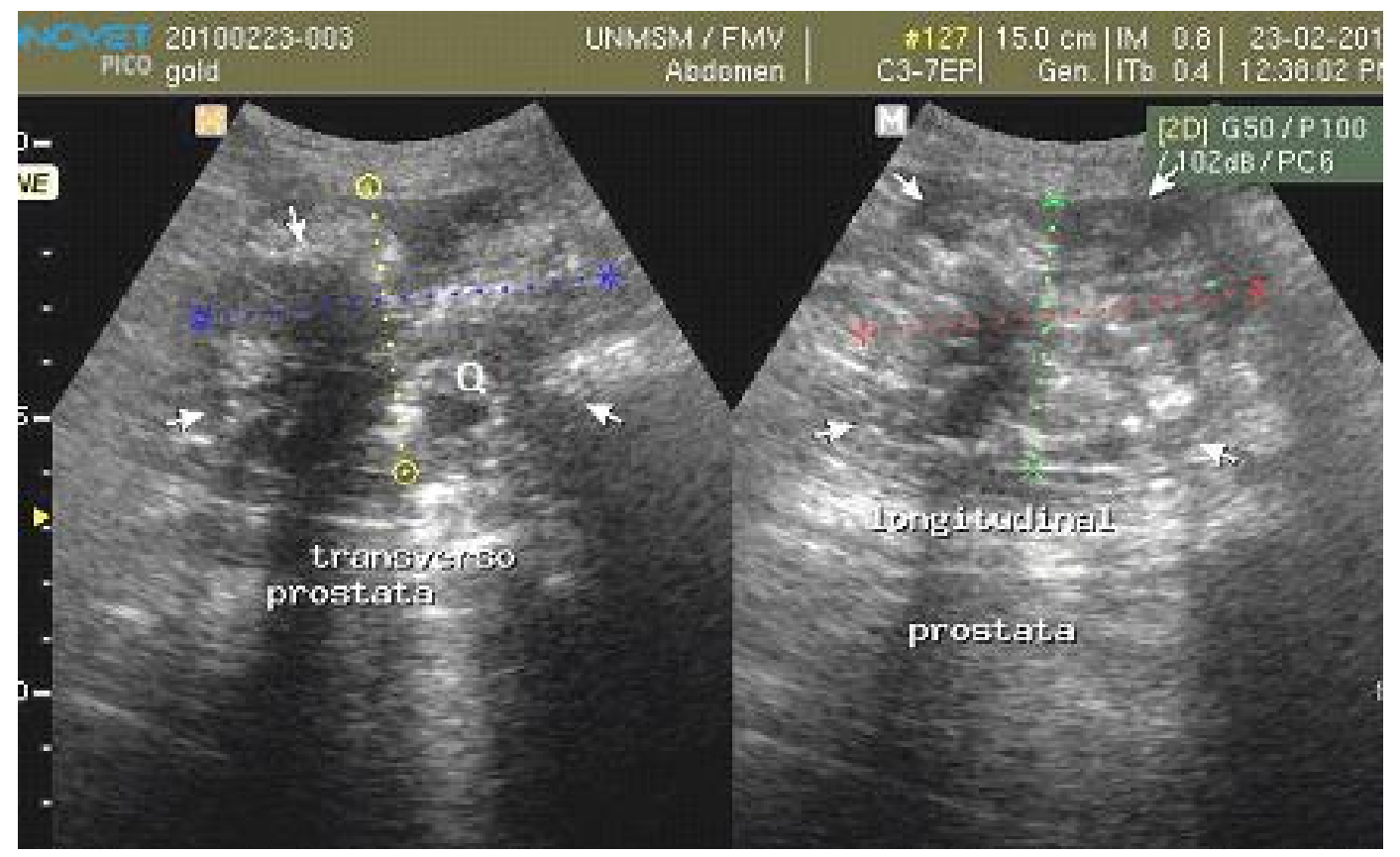

Figura 2. Lesiones compatibles con neoplasia prostática en un canino. Se observa parénquima heterogéneo, estructuras cavitarias de bordes irregulares $(\mathrm{Q})$, calcificaciones en zona craneal y caudal, áreas hiperecogénicas irregulares. 


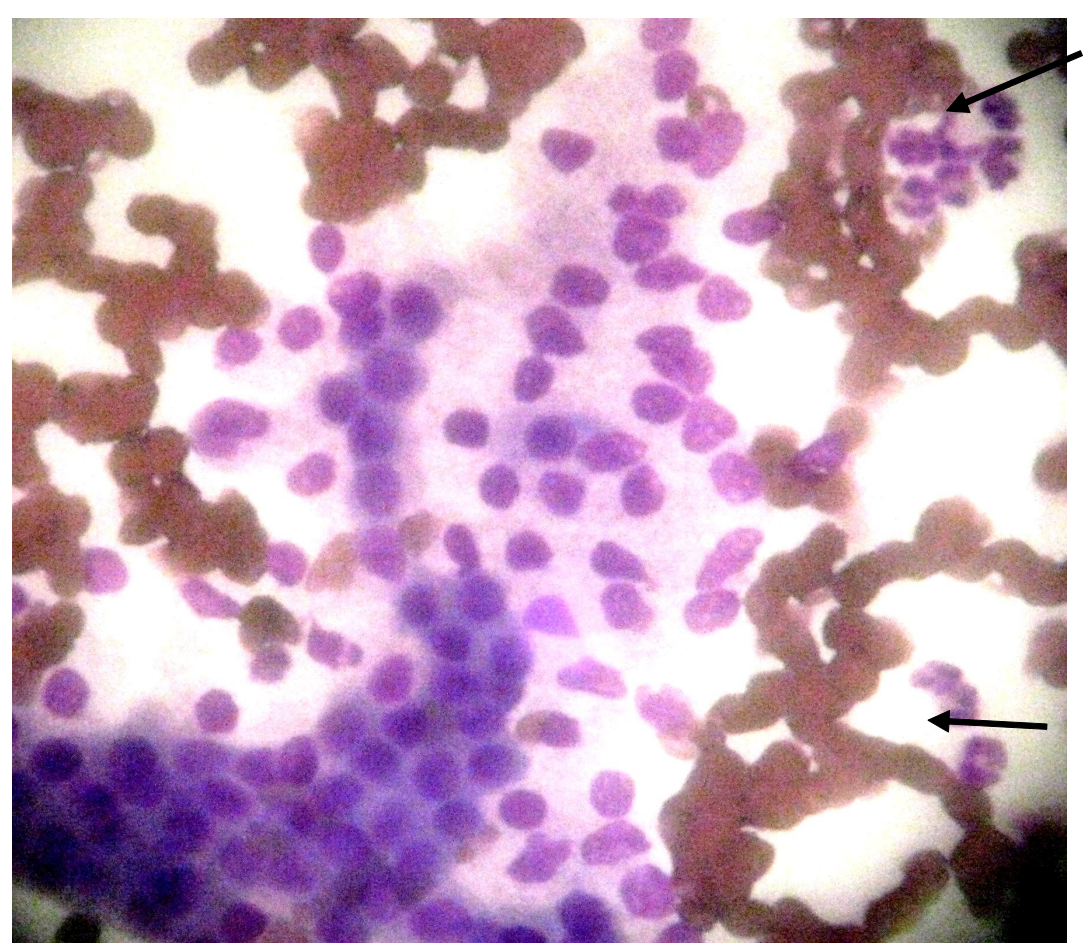

Figura 3. Citología exfoliativa de hiperplasia asociada a proceso inflamatorio. Células epiteliales prostáticas de apariencia normal obtenidas mediante aspirado de una próstata aumentada de tamaño. Las células son homogéneas, agrupadas a manera de acino conformada por un mayor número y neutrófilos (flechas). T-15. 400X

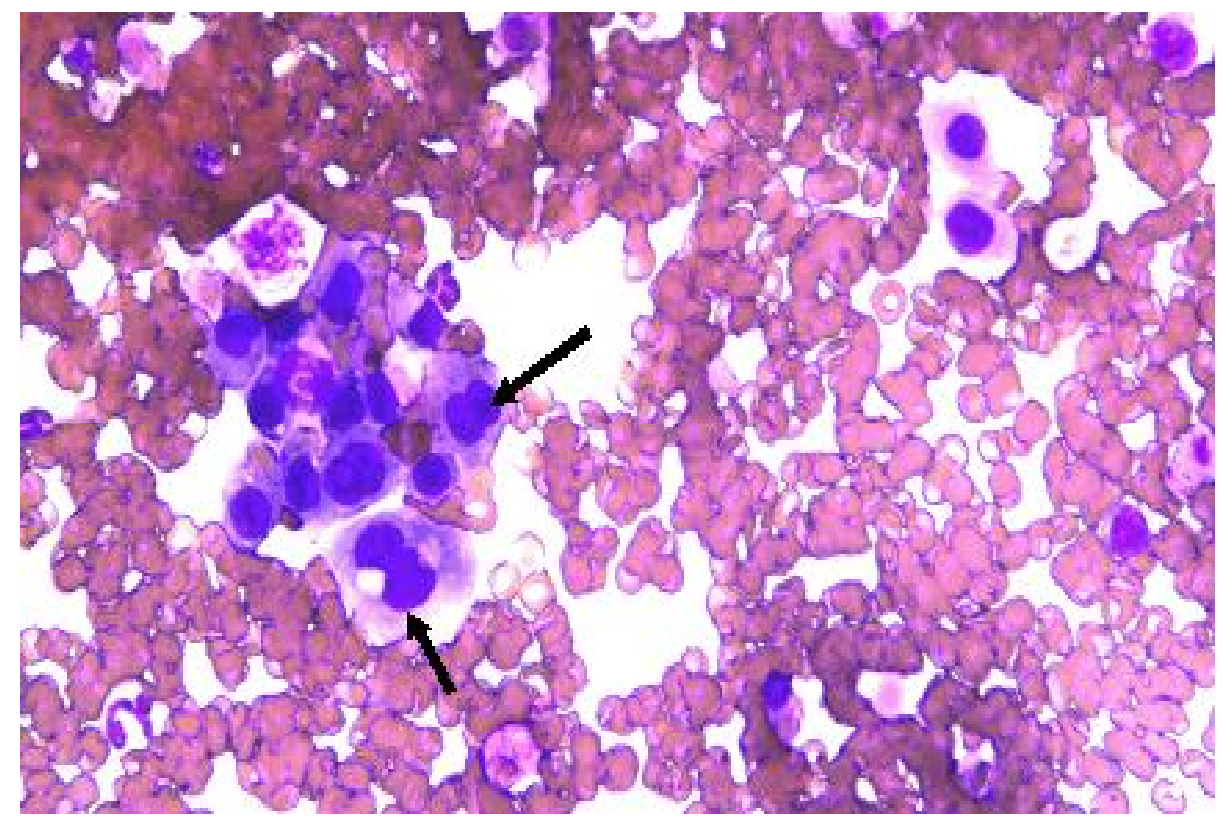

Figura 4. Citología exfoliativa compatible con adenocarcinoma prostático obtenido mediante aspirado. Células epiteliales prostáticas basófilas, atípicas, de diferentes tamaños, con núcleos prominentes y grandes, de tamaño y número variado (flechas), y con varios nucléolos. T-15. 400X 
en los que se observó un aumento del número de células prostáticas homogéneas asociado a la presencia de neutrófilos y eritrocitos rodeando los acinos prostáticos, observándose linfocitos y macrófagos en tres de los casos (Fig. 3).

En los pacientes con prostatitis se observaron neutrófilos en varios grados de degeneración y eritrocitos alrededor de las células prostáticas. Por otro lado, uno de los pacientes con lesiones compatibles con adenocarcinoma prostático fue un canino mestizo de cuatro años, en el que se observó células prostáticas con dos a cuatro núcleos ovalados de diferente tamaño y forma, algunas con núcleo gigante, pérdida de la relación núcleo/citoplasma y cromatina densa, figuras de mitosis, neutrófilos en moderada cantidad y eritrocitos abundantes. El otro caso de posible adenocarcinoma prostático se trató de un canino Pastor Alemán de 16 años, donde las células epiteliales prostáticas eran heterogéneas, con presencia de anisocitosis y anisocariosis, con núcleos gigantes y varios nucléolos, figuras de mitosis y neutrófilos en moderada cantidad (Fig. 4).

Se encontró concordancia entre los diagnósticos resultantes de la ultrasonografía y citología por aspiración guiada $(\mathrm{p}<0.05)$, siendo menor en el caso de prostatitis $(\mathrm{k}=0.315)$.

\section{DiscuSIón}

La próstata es un órgano relativamente sencillo de visualizar en una exploración ecográfica, la cual proporciona información valiosa para elaborar un pronóstico y administrar un tratamiento adecuado. En el presente trabajo, la evaluación previa al estudio citológico fue de gran utilidad para la evaluación del parénquima y la detección de anormalidades locales o difusas para su posterior aspiración.
El diagnóstico por los métodos de ultrasonografía y citología por aspiración mostraron una alta frecuencia de alteraciones prostáticas $(64.5 \%)$, donde la hiperplasia fue la patología de mayor frecuencia (32.3\%). El aumento de tamaño glandular prostático es un proceso que se presente generalmente en el perro adulto no castrado (Ravelo, 2000; Gadelha et al., 2009). Se puede afirmar entonces que la hiperplasia prostática es un proceso asociado al envejecimiento, que en mayor o menor grado está presente en la mayoría de caninos mayores de cinco años.

Los resultados indican que la edad es la variable que influencia la presentación de alteraciones prostáticas, obteniéndose resultados similares al estudio de Barsanti y Finco (2002). La próstata presenta tres periodos de desarrollo. El primer periodo es el de embriogénesis y el desarrollo posnatal inmediato que finaliza cuando el animal tiene de dos a tres años. La segunda consiste en la fase de desarrollo hipertrófico exponencial que es dependiente de andrógenos y termina cuando el animal tiene de 12 a 15 años (Berry e Isaacs, 1984; Verstegen, 1999), de allí que los perros presentan cierto grado de hiperplasia prostática a partir de los cinco años de edad (Avendaño, 2008). La última etapa es la involución senil que se inicia cuando disminuye la producción de andrógenos (Berry e Isaacs, 1984; Verstegen, 1999). En el presente estudio se pudo corroborar esta información ya que hubo una gran diferencia entre las frecuencias de presentación de acuerdo a la edad, donde el $58 \%$ de los animales de 5 a 10 años presentaban alteraciones prostáticas en comparación con el $100 \%$ a partir de los 10 años de edad.

Cabe resaltar que un canino de cuatro años diagnosticado como proceso inflamatorio mediante ultrasonografía dio al estudio citológico un cuadro compatible con neoplasia. Desde el punto de vista ecográfico, es difícil distinguir un proceso neoplásico de la prostatitis dado que las características de 
ecogenicidad pueden ser similares (afectación difusa, mineralización, distorsión parenquimatosa); por eso, la biopsia o citología debe ser de curso obligatorio para determinar el diagnóstico definitivo (Liste, 1997).

El diagnóstico histopatológico de las alteraciones prostáticas representa la «prueba de oro» porque la arquitectura del tejido y el detalle celular son evaluados (González et al., 2010); sin embargo, la citología tiene varias ventajas sobre la histopatología en la investigación de la enfermedad prostática, ya que es una técnica menos invasiva, que en muchas ocasiones no requiere de anestesia general y los resultados son obtenidos en corto tiempo (Powe et al., 2004). Asimismo, estas ventajas permiten que la citología sea una prueba de mayor aproximación al diagnóstico que la ultrasonografía.

La punción de prostatitis supurativas o con abscesos está contraindicada, porque las bacterias pueden quedar sembradas a lo largo del trayecto de la aguja y producir una infección moderada a severa (Barsanti y Finco, 2002; González et al., 2010). No obstante, en algunos casos se podría diferenciar las cavidades prostáticas por las características de su contenido en la imagen ecográfica, aunque a veces, la próstata presenta solamente estructuras quísticas sin otra alteración anatómica. En estos casos, se debería realizar la aspiración guiada para evaluar el líquido obtenido (Paclikova et al., 2007), pudiendo remitirse la muestra para un cultivo bacteriológico, y poder dar el tratamiento antibiótico adecuado al paciente (Liste, 1997).

El riesgo del desarrollo de complicaciones por la aspiración es relativamente bajo; sin embargo, puede presentarse hematuria, peritonitis por contaminación peritoneal por la aspiración o daño iatrogénico de la uretra (Paclikova et al., 2006). Debido a esto, es necesario realizar la asepsia del lugar a punzar y guiar la aguja mediante la imagen ecográfica. En este estudio no se obtuvo ninguna complicación por la aspiración eco-guiada.

\section{Conclusiones}

- El $64.5 \%$ de los canes presentaron patologías prostáticas diagnosticadas por ultrasonografía y citología, donde la hiperplasia fue la alteración más frecuente.

- Las alteraciones prostáticas se presentaron en el $58.6 \%$ de canes mayores de cinco años y en todos los canes mayores de 10 años.

- La citología se destaca por ser una prueba factible y rápida, que puede ser realizada por el Médico Veterinario clínico para un tratamiento más eficaz en un corto periodo de tiempo.

\section{LitTeratura Cittada}

1. Avendaño A. 2008. Evidencia de alteraciones prostáticas anatómicas detectadas por ultrasonografía transabdominal en caninos adultos enteros aparentemente sanos. Tesis de Médico Veterinario Zootecnista. Lima: Univ Peruana Cayetano Heredia. 29 p.

2. Barsanti J, Finco D. 2002. Enfermedades prostáticas caninas. En: Ettinger S, Feldman E (eds). Tratado de medicina interna veterinaria. $6^{\mathrm{a}}$ ed. España: Elsevier. p 1952-1971.

3. Berry S, Isaacs J. 1984. Comparative aspects of prostatic growth and androgen metabolism with aging in the dog versus the rat. Endocrinology 114: 511-520.

4. Gadelha C, Vicente W, Ribeiro A, Apparicio M, Covizzi G Machado L. 2009. Age-related ultrasonography, cytology, and microbiologic exam of canine prostate. Arq Bras Med Vet Zootec 61: 1261-1267.

5. García A. 1996. Fisiología veterinaria. España: Interamericana McGraw Hill. $1074 \mathrm{p}$.

6. González G, Maffrand C, Guendulain C, Cafaratti M, Galetto M, Gobello C. 2010. Sensibilidad y especificidad de la 
aspiración guiada por ecografía para el diagnóstico de hiperplasia prostática y prostatitis crónica canina. Revista Científica FCV-LUZ 20: 617-622.

7. Liste F. 1997. Hallazgos ecográficos en diferentes afecciones de la próstata en el perro: descripción de 8 casos clínicos. Clín Vet Pequeños Animales 17(2): 114-128.

8. Meinkoth J, Cowell R, Tyler R, Morton $R$. 2009. Recogida y preparación de muestras. En: Cowell R, Tyler R, Meinkoth J, DeNicola D (eds). Diagnóstico citológico y hematológico del perro y el gato. $3^{\mathrm{a}}$ ed. España: Elsevier. p 1-11.

9. Miguel de Priego C. 2007. Correlación entre el tamaño ecográfico de la glándula prostática y el riñón izquierdo en caninos. Tesis de Médico Veterinario Zootecnista. Lima: Univ Peruana Cayetano Heredia. 27 p.

10. Nyland T, Mattoon J. 2002. Diagnóstico ecográfico en pequeños animales. $2^{\mathrm{a}}$ ed. España: Multimédica. $490 \mathrm{p}$.

11. Paclikova K, Kohout P, Vlašin M. 2006. Diagnostic possibilities in the management of canine prostatic disorders. Vet Med-Czech 51(1): 1-13.

12. Paclikova K, Kohout P, Vlašin M. 2007. Catheter biopsy as a useful tool to establish an early diagnosis for canine prostatic disorders. Acta Vet Brno 76: 475-485.

13. Powe J, Canfield P, Martin P. 2004. Evaluation of the cytologic diagnosis of canine prostatic disorders. Vet Clin Pathol 33: 150-154.

14. Ravelo D. 2000. Características histopatológicas de la glándula prostática en caninos mayores de cinco años. Tesis de Médico Veterinario. Lima: Univ Nac. Mayor de San Marcos. 41 p.

15. Senior D. 2006. Alteraciones urinarias. En: Schaer M (ed). Medicina clínica del perro y el gato. España: Masson. p 445449.

16. Verstergen J. 1999. Alteraciones reproductivas del macho. En: Simpson GM, Gary CW, England GC, Harvey SM (eds). Manual de reproducción y neonatología en pequeños animales. Madrid: Hartcourt. p 99-102. 